
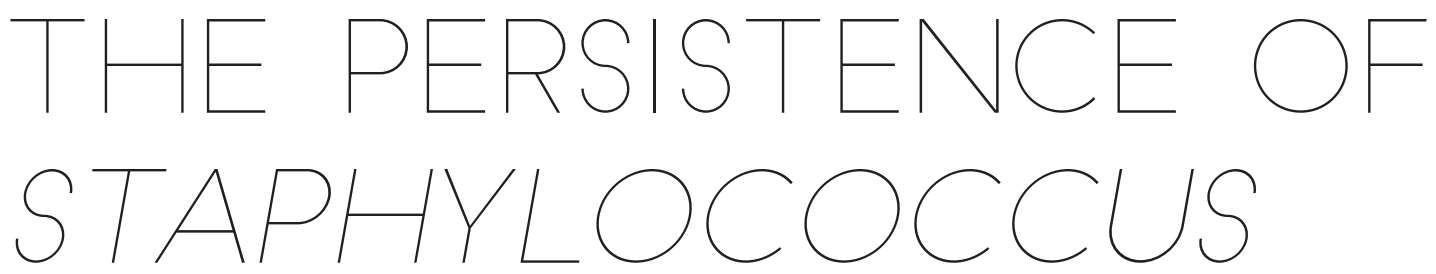

$\Lambda \mathrm{W}+\mathrm{C}$
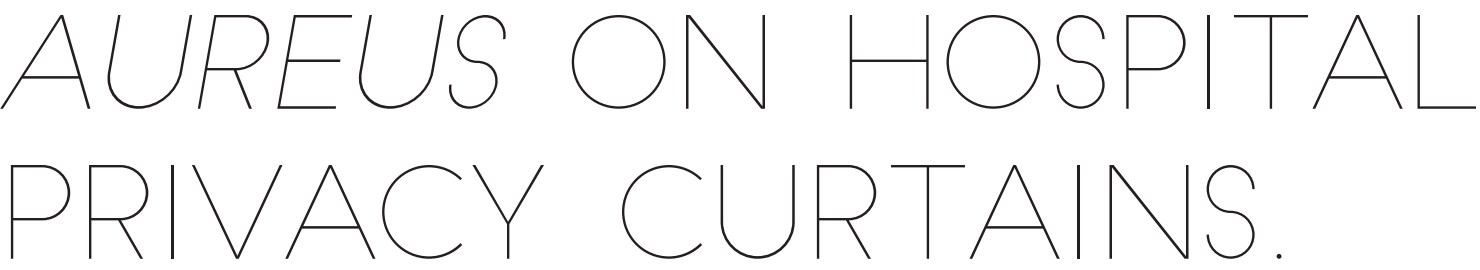

Sarah E. Cole Brittany J. Gasper*

'Current address - 11200 SW 8 ${ }^{\text {th }}$ St, Miami, FL 33199

* Center for Teaching and Learning, Kent State University, Kent, Ohio

Manuscript recieved 19 March 2019; accepted 30 July 2019 
54 • Fine Focus, Vol 52019

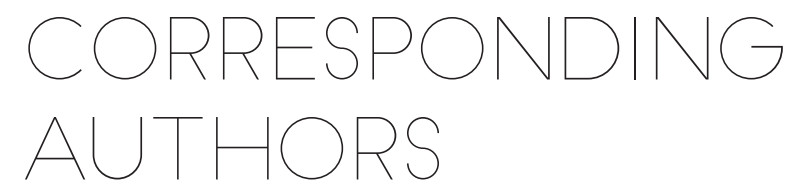

Sarah E. Cole

sarahecole95@yahoo.com

Brittany J. Gasper
bgasper@flsouthern.edu

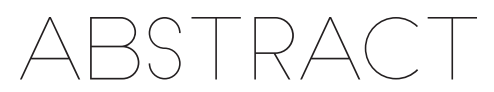

While healthcare professionals are working in hospitals, they will often manipulate the privacy curtains during the care of their patients. Studies have shown that the transfer of bacteria from hands to the curtains and vice versa is possible. Despite the possibility of hospital curtains being a mode of infection transmission, studies have shown that $53 \%$ of hospitals surveyed did not have a policy for cleaning or changing their curtains. The question that this study focused on was whether curtain material affects the persistence of Staphylococcus aureus. In this study, five different curtain types were inoculated with overnight, diluted, and finger imprint cultures of $S$. aureus. They were incubated at room temperature and were sampled for growth regularly onto Mannitol Salt Agar plates. The colonies were counted, and one-way ANOVA statistical analysis was completed on the data. The statistical analysis showed that the length of persistence of liquid cultures of $S$. aureus on the curtains was not dependent upon initial concentration. Finger imprint inoculations of four curtain varieties had statistically significant longer persistence times than the liquid cultures. Only the curtain type composed of 100\% antimicrobial polyester with water repellant had significantly lower persistence times for the finger imprint culture than the other four curtains. The results suggest that the 100\% inherently flame resistant antimicrobial polyester curtain material reduces $S$. aureus persistence times and that it may benefit hospitals to use this type of curtain.

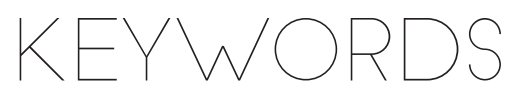

- persistence

- health-care

- associated infection

- nosocomial infection

- hospital curtain

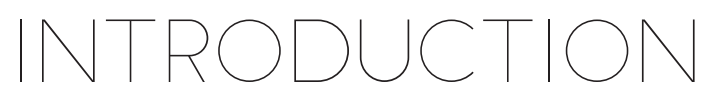

The environment of the hospital has an important role in the transmission of health-care associated pathogens which cause nosocomial infections. Studies have found that hospital privacy curtains are quickly contaminated by microorganisms that can be transferred to the hands of healthcare workers, the patient, and the surrounding environment $(21,30)$. In 1988, there was an outbreak of multiple-antibiotic-resistant Acinteobacter baumannii in the Queen Elizabeth Hospital in Birmingham, UK (7). After an investigation, carbapenem-resistant $A$. baumannii was isolated from surfaces of equipment, beds, mops, and curtains. It was determined that the curtains were the main source of the outbreak because they had the highest number of the organism present on them (7).

In addition to the curtains being easily contaminated with pathogens that can be transferred via the hands to people or other surfaces, some bacteria can persist on hospital surfaces, including curtains, for weeks and even months $(15,19,20)$. Persistence in this case refers to the ability of an organism to survive and be cultured from a surface (15). A study by DeAngelis and Khakoo (8) indicated that these lengthy persistence times are problematic. Their study included surveys completed by hospitals on curtain cleaning policies. Over half of the hospitals surveyed (53\%) said they do not have a policy for cleaning the hospital curtains, 37\% said they only clean their curtains when visibly soiled, $13 \%$ clean the curtains every year, 13\% clean their curtains every three months, and only 13\% clean their curtains every month. In other words, the most frequently some hospitals change their curtains is every month. Since only $13 \%$ of the total hospitals surveyed clean their curtains that often, this means most of the hospitals either do not have a cleaning policy or change the curtains every three months or greater. Mitchell et al. (2015) noted that the attention 
put into cleaning porous, soft surfaces such as privacy curtains and room furnishings is far less than that given towards disinfection of porous surfaces (18). As there are nosocomial pathogens such as Staphylococcus aureus, Klebsiella quasipneumoniae subsp. similipneumoniae (formerly known as K. pneumoniae) (10), and Candida albicans that can last on hospital surfaces for longer than four months (15), this three month cleaning interval may not be frequent enough. This suggests there needs to be more frequent cleaning and a change of policy.

Despite the importance of the topic, there has not been a large amount of research completed on the topic of hospital curtains and bacteria or bacterial infections. The studies completed so far have varied substantially in their inoculation technique and their analytical methods. Some have taken finger imprints from curtains actively hanging in hospital rooms ( 3 , $14,16,17,21,22,24)$. Others have inoculated known concentrations of bacteria onto specified areas of curtain swatches $(13,27)$ or hospital fabric $(20)$. These studies have also differed in duration from as short as seven days (17) to as long as six months $(3,14,25)$. There are different varieties of hospital curtains available including those containing antimicrobial fabric and some coated with water repellant. However, most studies only used one type of curtain and this varied from vinyl (21) to flame resistant (FR) (22), propylene (14), disposable sporicidal (13), and polyester (16, $24)$, amongst others. Only two studies $(20,24)$ were shown to compare more than one type of curtain, and only three studies used statistical tests to analyze their results $(3,17,24)$. Contradictory results of previous studies include some that have showed a high rate of Methicillin Resistant S. aureus (MRSA) on curtains (21) and those that have not (16). Kurashige (2016) mentions that curtain type may influence these results and should be further examined.

This study intended to expand on the existing research. The focus was on $S$. aureus, a species of pathogenic bacteria that causes nosocomial infections and has been shown to be capable of developing antibiotic resistance to become hospital-associated MRSA (9). This study combined aspects of previous findings by measuring the persistence times of $S$. aureus inoculated both directly with a known concentration or by fingerprint on five different curtain types. These curtain types included three different combinations of FR and non-FR polyester with no antimicrobial properties and two antimicrobial polyester curtains, one with and one without water repellant. We hypothesize that the composition of the different curtain types, spe-
Staphylococci on hospital curtains • 55 cifically the presence of antimicrobial properties, will influence the persistence of $S$. aureus.

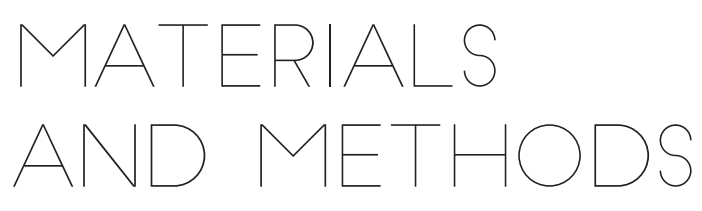

\section{Curtain Varieties and Codes}

Five different curtain varieties were used in this study. Each curtain was given a code for ease of designation and to prevent bias on the part of the experimenter when counting colony-forming units (CFUs) and persistence times. The curtain varieties and codes used in this study can be found in Table 1.

\begin{tabular}{|c|l|}
\hline Curtain Code & \multicolumn{1}{|c|}{ Curtain Material } \\
\hline A & $\begin{array}{l}46 \% \text { FR polyester, 27\% post-consumer recycled polyester, and 27\% post- } \\
\text { industrial recycled polyester }\end{array}$ \\
\hline B & $87 \%$ polyester, 13\% Avora FR polyester \\
\hline C & $52 \%$ post-consumer recycled FR polyester, 48\% FR polyester \\
\hline D & $56.6 \%$ Amy antimicrobial polyester, 43.4\% recycled FR polyester \\
\hline E & $100 \%$ inherently FR antimicrobial polyester with water repellant \\
\hline
\end{tabular}

Table 1: Composition and codes of the five different curtains used in this study.

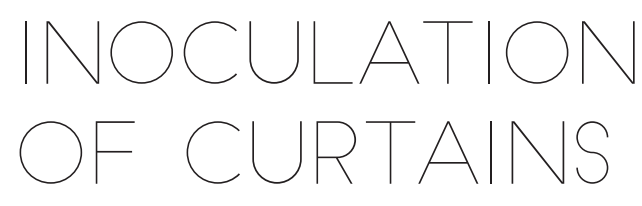

Each curtain variety was cut into $2 \times 2 \mathrm{~cm}$ squares that were sterilized by autoclaving. Sterile curtain squares were inoculated with $S$. aureus by three different methods. For each method, six squares of each curtain type were inoculated. All overnight $(\mathrm{O} / \mathrm{N})$ cultures of $S$. aureus (ATCC 12600) were grown in liquid tryptic soy broth (TSB) media at $37^{\circ} \mathrm{C}$ for 24 hours.

Method one (Concentrated liquid culture technique) - Curtain squares were inoculated with 20 $\mu \mathrm{l}$ of a liquid $\mathrm{O} / \mathrm{N}$ culture of $S$. aureus. This was the most concentrated solution inoculated and was intended to simulate an extreme contamination event such as 


\section{6 • Fine Focus, Vol 52019}

direct contact of a contaminated fluid sample with the curtain. Measurement of the OD600 on a Genesys $10 \mathrm{~S}$ UV-Vis spectrophotometer (Thermo Scientific) indicated this was equivalent to a total bacterial load of $2.0 \times 107 \mathrm{cfu}$ or a density of $5.0 \times 106 \mathrm{cfu} / \mathrm{cm} 2$. Method two (Diluted liquid culture technique) - Curtain squares were inoculated with $20 \mu \mathrm{l}$ of a liquid $\mathrm{O} / \mathrm{N}$ culture of $S$. aureus diluted to an OD600 of 0.08 in a sterile $0.9 \%(\mathrm{w} / \mathrm{v})$ sodium chloride solution. This was equivalent to a total bacteria load of $1.3 \times 106$ cfu or a density of $2.7 \times 104 \mathrm{cfu} / \mathrm{cm} 2$. This OD600 was chosen as the diluted culture standard as it closely represents the ODᄀ600 value of the $0.5 \mathrm{McF}$ arland standard used for inoculated samples during antimicrobial susceptibility testing (6). A diluted sample was included to see if the persistence of $S$. aureus was dependent on the initial concentration inoculated and to represent direct contact between curtains and diluted fluid samples. Method three (Colony finger imprint technique) - Curtain squares were inoculated with a colony of $S$. aureus by touching the colony with a gloved finger and pressing it onto the curtain piece, making sure to equally touch each area of the curtain. The colonies of $S$. aureus used for this step were similar in size and obtained from a quadrant streaked mannitol salt agar (MSA) plate that was incubated at $37^{\circ} \mathrm{C}$ for 48 hours. Curtain E was the only curtain where the overnight and diluted cultures were not inoculated onto the curtain. Only the finger imprint technique was used to inoculate this curtain because it is water repellant and was not able to absorb the liquid cultures. After the curtain squares were inoculated, they were left to dry in separate sterile petri dishes for 20 minutes.

\section{MEASUREMENT

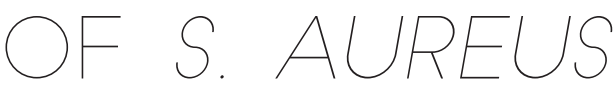 \\ PRESISTENCE}

After inoculation and drying, an initial measure of bacterial growth was aseptically taken from each curtain square. A flame sterilized pair of tweezers was used to hold the curtain square steady while a sterile cotton swab dipped in a sterile $0.9 \%(\mathrm{w} / \mathrm{v})$ sodium chloride solution was used to evenly sample each curtain with 10 strokes (five down and five up). This cotton swab was then used to evenly, continuously streak onto an MSA plate that was incubated at $37^{\circ} \mathrm{C}$ for 48 hours. After incubation, the number of CFU on each plate was counted. If more than $300 \mathrm{CFU}$ were present on the plate, it was recorded as too numerous to count. Curtain squares were maintained at room temperature in sterile lidded petri dishes to represent the equivalent environment of a hospital setting. Swabbed samples of $S$. aureus were taken from the squares at a maximum of three day intervals until growth of five CFU or less was seen on an MSA plate incubated at $37^{\circ} \mathrm{C}$ for 48 hours for two consecutive samplings. To conserve resources, this was modified from Neely and Maley (2000), who sampled until no CFU were seen for two consecutive samplings, as five or fewer CFU was considered sufficient decline to end the measurement of persistence. Once all of the data were collected, the number of days that the $S$. aureus persisted on each sample of each curtain type was counted. The day that the curtain pieces were inoculated and initially swabbed was considered day zero. All of this data were compiled and analyzed using one-way ANOVA statistics with the Tukey honestly significant difference test.

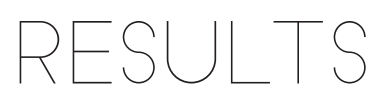

The persistence time of $S$. aureus was measured (in days) and compared for the three different inoculation methods on curtain varieties A, B, C, and D. The comparisons of the mean persistence times from the three inoculation techniques for each type of curtain are shown in Fig. 1.

All curtain varieties in Fig. 1 showed significantly longer persistence times after inoculation of a colony using the finger imprint technique compared to inoculation of a concentrated or diluted $\mathrm{O} / \mathrm{N}$ liquid culture. Curtains A, B, and C showed a p-value of $<0.0001$ while the $\mathrm{p}$-value for $\mathrm{D}$ was less than 0.05 . There was no significant difference in the persistence times between inoculation of a concentrated or diluted $\mathrm{O} / \mathrm{N}$ culture for any of these curtain types.

The mean persistence times for $S$. aureus inoculated using concentrated $\mathrm{O} / \mathrm{N}$ liquid cultures and diluted liquid cultures was compared for the four curtain varieties $(\mathrm{A}, \mathrm{B}, \mathrm{C}$, and $\mathrm{D})$. These mean persistence times are shown in Figs. 2 and 3, respectively. The persistence time of $S$. aureus on curtain $C$ was significantly longer than the other curtain types (p-value < 0.01 ). The mean persistence time for curtain varieties $\mathrm{A}, \mathrm{B}$, and $\mathrm{D}$ ranged from 7.76 to 10.0 days while the 
mean persistence time for curtain C was 14.5 days. Interestingly, there was no statistically significant difference between the mean persistence times of $S$. aureus on these four curtain varieties when inoculated with a diluted $\mathrm{O} / \mathrm{N}$ culture. The mean persistence times ranged from 9.83 to 13.3 days.

A comparison of the mean persistence times for $S$. aureus inoculated using the colony finger imprint technique on all curtain varieties is shown in Fig. 4. The mean persistence time of $S$. aureus inoculated onto curtain variety $\mathrm{E}$ was significantly less than the four other curtain varieties ( $\mathrm{p}$-value $<0.0001)$. The mean persistence time for the four other curtain varieties ranged from 22.0 days to 27.0 days while it was only 4.0 days for curtain E.

There was no significant difference in the number of $S$. aureus CFUs recovered over time from each curtain type (data not shown). Regardless of the inoculation method, all curtain types A-E showed an initial high number of CFUs that decreased at a steady pace before the bacteria were no longer able to persist. Great variation independent of curtain type was seen in the specific numbers of CFUs at any given time point, but the general decline was consistent.

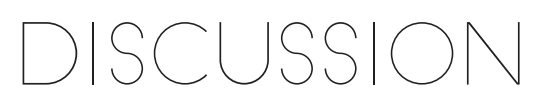

When comparing the mean persistence times of the $\mathrm{O} / \mathrm{N}$ and diluted cultures of each of the curtains $\mathrm{A}, \mathrm{B}, \mathrm{C}$, and $\mathrm{D}$, there was no difference between the $\mathrm{O} / \mathrm{N}$ and diluted cultures. In other words, the persistence following liquid inoculation was not dependent on initial concentration. On the other hand, there was a statistically significant difference between mean persistence times of the finger imprint cultures and both the overnight and diluted cultures for all of these curtains. In addition, mean persistence time of $S$. aureus inoculated by the finger imprint lasted much longer than the other cultures curtain varieties $\mathrm{A}, \mathrm{B}$, $\mathrm{C}$, and $\mathrm{D}$, implying that the finger imprint inoculation method did have an effect on persistence times, lasting more than 10 days longer than the $\mathrm{O} / \mathrm{N}$ or diluted cultures. There was no significant difference in the initial CFU counts for any of the inoculation methods (data not shown). These data suggested that the solid inoculation of a bacterial colony increased the persistence of $S$. aureus compared to a liquid inoculation.
Staphylococci on hospital curtains • 57

To our knowledge, no research comparing the persistence times of bacteria inoculated from both liquid and solid cultures has been published. Kotsanas et al. (2012) inoculated curtain samples with liquid cultures and took finger imprints at specified time points to culture from the curtain and measure persistence (13). They found $S$. aureus to persist for 10 days but show no growth at 2 months. This is consistent with the range of mean persistence times of 8.50 to 14.5 days we measured from our liquid inoculations. Interestingly, they found < 10 CFU after one day of incubation when culturing by the finger imprint method and incubating at $37^{\circ} \mathrm{C}$ on horse blood agar, an enriched media (11). By comparison, our CFU values from curtains incubated for one day following liquid inoculation and cultured by swabbing and incubating at $37^{\circ} \mathrm{C}$ on MSA were consistently higher than $10 \mathrm{CFUs}$ for all curtain types (data not shown). The counts ranged from a low of 13 CFUs to a countable high of 123 CFUs. Of the 33 samples for which the CFU count after one day is available, 15 were found to be too numerous to count. The curtains in Korvath et al. (2012) were inoculated with 4 x $105 \mathrm{CFU} / \mathrm{cm} 2$ of culture, comparable to the $5.0 \times 106$ and $2.7 \times 104 \mathrm{CFU} /$ $\mathrm{cm} 2$ in the $\mathrm{O} / \mathrm{N}$ and diluted samples, respectively. These data suggested the swabbing technique may be a more thorough method for culturing from the entire curtain sample than taking a finger imprint from the center of the curtain (13).

With the comparison of the persistence time from $S$. aureus inoculated from concentrated $\mathrm{O} / \mathrm{N}$ cultures on curtains $\mathrm{A}, \mathrm{B}, \mathrm{C}$, and $\mathrm{D}$, there was statistical significance between curtain $C$ and A, B, and D $(p<0.01)$. C had the longest persistence time of all of the overnight cultures with an average of 14.5 days as opposed to the averages of $8.50,10.0$, and 7.67 days for curtains A, B, and D, respectively. This statistical significance suggested that the curtain material of $\mathrm{C}$ had an effect on the persistence of the $S$. aureus concentrated $\mathrm{O} / \mathrm{N}$ culture. Interestingly, these results were not reproducible when comparing persistence of $S$. aureus grown from diluted $\mathrm{O} / \mathrm{N}$ cultures. This suggests a possible concentration-dependency on the persistence of $S$. aureus on this particular curtain material though the lack of statistical difference between the persistence of $S$. aureus from concentrated and diluted $\mathrm{O} / \mathrm{N}$ cultures would conflict this. Additional research is needed to address these conflicting results.

The main distinction in curtain material between curtain $\mathrm{C}$ and the others is that it had the highest percentage of post-consumer recycled FR polyester 
58 • Fine Focus, Vol 52019

Figure 1
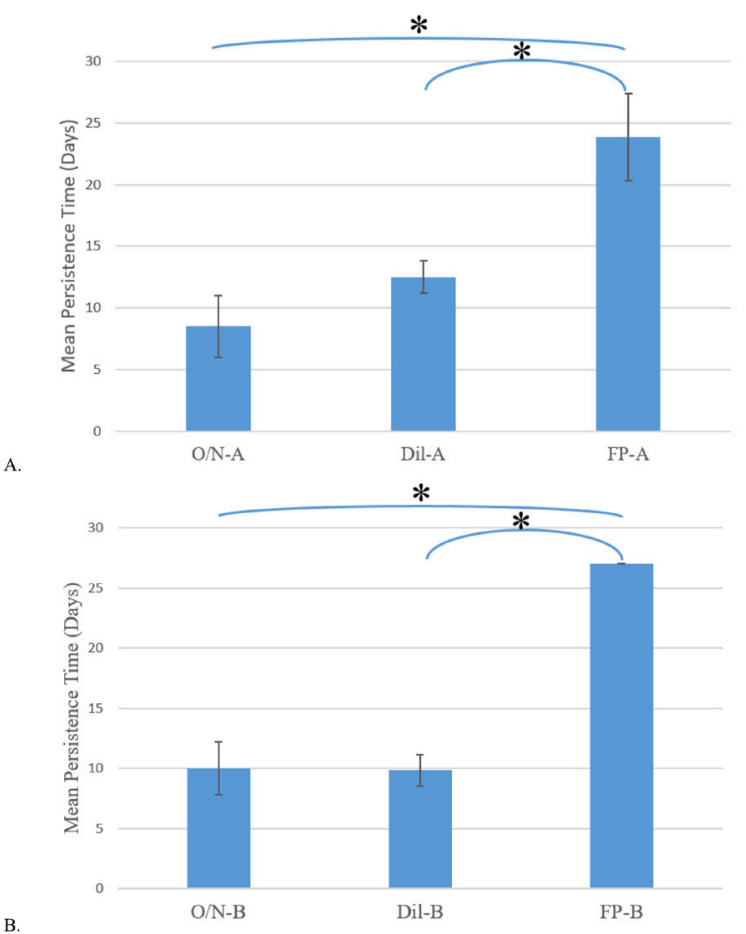

Figure 1: Mean Persistence Times of $S$. aureus from Different Inoculation of Techniques:

The mean persistence times in days for curtain varieties A (Panel A), B (Panel B), C (Panel C), and D (Panel D) are shown after inoculation by concentrated $\mathrm{O} / \mathrm{N}$ culture, diluted $\mathrm{O} / \mathrm{N}$ culture (Dil), or colony finger imprint technique (FP). The $\mathrm{O} / \mathrm{N}$ and $\mathrm{D}$ cultures contained $2.0 \times 107$ and $1.3 \times 106$ CFU, respectively. Each bar represents the mean persistence time \pm standard deviation of $n=6$ inoculated samples. Statistical analysis was completed using a one-way ANOVA ( indicates statistical significance with a p-value $<0.05)$.

Figure 2

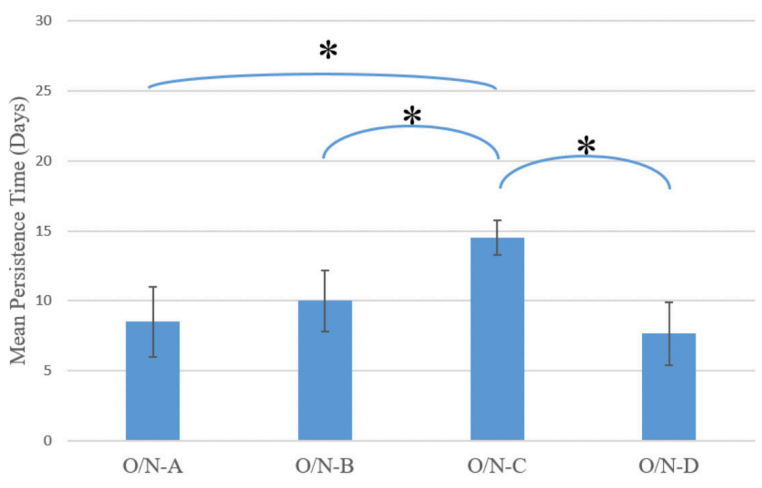

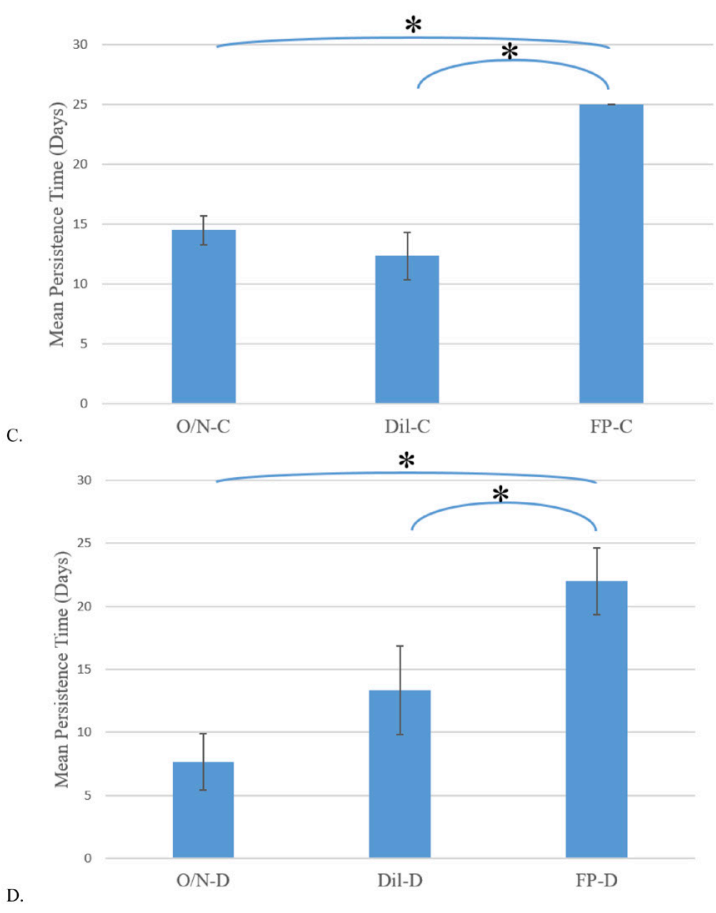

D. 


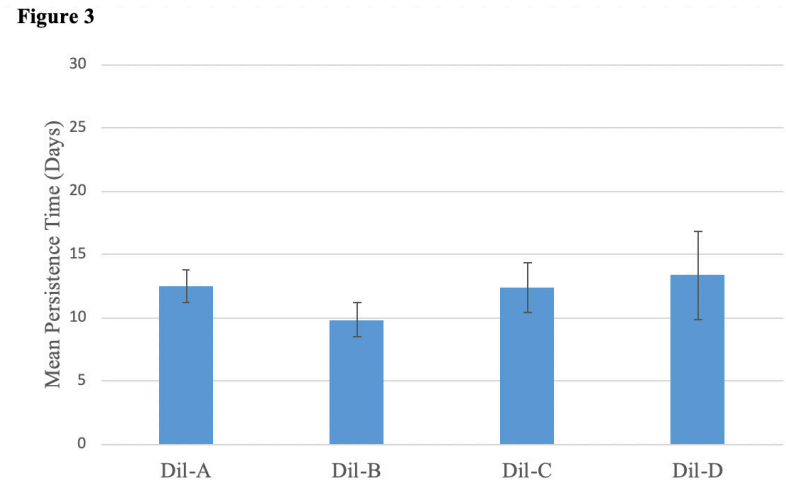

Figure 3 - Mean Persistence Times of $S$. aureus Inoculated from a Diluted Liquid $\mathrm{O} / \mathrm{N}$ Culture:

The mean persistence times in days for curtain varieties $A$, $\mathrm{B}, \mathrm{C}$, and D inoculated with a diluted (Dil) $\mathrm{O} / \mathrm{N}$ culture of $S$. aureus are shown. The D culture contained $1.3 \times 106$ CFU. Each bar represents the mean persistence time \pm standard deviation of $\mathrm{n}=6$ inoculated samples. Statistical analysis was completed using a one-way ANOVA.

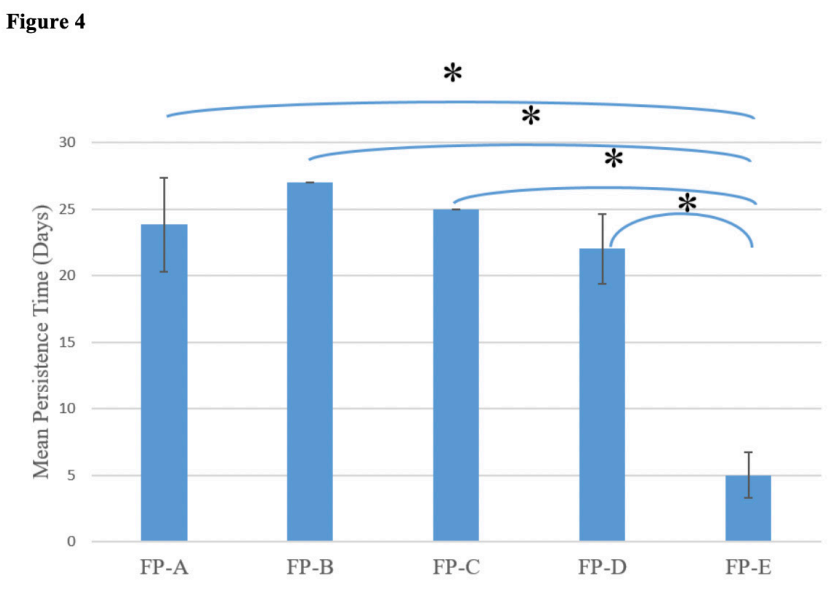

Figure 4 - Mean Persistence Times of S. aureus Inoculated by Finger Imprint of a Bacterial Colony: The mean persistence times in days for curtain varieties $\mathrm{A}, \mathrm{B}, \mathrm{C}, \mathrm{D}$, and $\mathrm{E}$ inoculated with a colony of $S$. aureus by the finger imprint technique (FP) are shown. Each bar represents the mean persistence time \pm standard deviation of $\mathrm{n}=6$ inoculated samples. Statistical analysis was completed using a one-way ANOVA ( $\star$ indicates statistical significance with a p-value $<0.05)$.
Staphylococci on hospital curtains • 59 at $52 \%$, higher than the $27 \%$ found in curtain $\mathrm{A}$, the only other curtain containing this material. Since curtain $\mathrm{A}$ is composed of the greatest percentage of total recycled polyester (54\% including post-consumer and post-industrial), it seems unlikely that recycled polyester alone could account for the longer persistence time seen with inoculation of $\mathrm{O} / \mathrm{N}$ cultures. Further research will compare the persistence times of $S$. aureus inoculated from a range of dilutions onto curtains $\mathrm{A}$, $\mathrm{B}$, and $\mathrm{C}$, composed of mixed recycled, no recycled, and post-consumer recycled polyester, respectively. Most studies have only used one type of curtain. Neely and Maley (2000) compared the persistence of Staphylococcus and Enterococcus on multiple types of hospital fabrics (20). Their values for persistence of methicillin sensitive $S$. aureus after liquid inoculation onto 100\% polyester range from 10 - 56 days, comparable on the lower end to the range of liquid culture persistence from this study. Compared to other fabrics, polyester and polyethylene provided the longest persistence times for $S$. aureus while inoculation onto $100 \%$ cotton, terry cloth, and a 60-40\% cotton-polyester blend resulted in comparably shorter persistence times (20).

While curtain varieties $A, B, C$, and D all showed increased persistence of $S$. aureus inoculated by the finger imprint method, curtain variety E had significantly decreased persistence times by comparison ( $\mathrm{p}<0.01)$. The mean persistence of $S$. aureus on curtain E (4.0 days) is more than 15 days shorter than the averages of the other four curtains, suggesting the material of this curtain did have an effect on the persistence time. Curtain $\mathrm{E}$ is the only variety to contain $100 \%$ inherently antimicrobial FR polyester with a water repellant. Curtain D also has antimicrobial polyester $(56.6 \%)$, but it does not contain a water repellant. Interestingly, the lower antimicrobial composition of curtain D did not seem to have an effect on persistence times as it showed no significant difference from curtains A or B that contain no antimicrobial composition. It is not clear if the significantly decreased persistence of curtain $\mathrm{E}$ is due to the 100\% antimicrobial polyester, the water repellant nature, or both. In order to determine how the water repellant affected the persistence times from the finger imprint inoculation, additional experimentation would need to be completed comparing 100\% FR antimicrobial polyester with water repellant materials to the same curtain material without the water repellant treatment. In addition, to determine how this curtain material affects the liquid cultures, experimentation would be 
60 - Fine Focus, Vol 52019

needed comparing the persistence times of curtains A, B, C, and D to the 100\% inherently FR antimicrobial polyester without water repellant.

These results have an important implication in the hospital setting because the finger imprint cultures are a better representation of the inoculation method that would take place in the hospital. For example, in the hospital, healthcare professionals might touch the source of infection with their gloved hands and then touch the curtain. It may benefit hospitals to use this type of high percentage antimicrobial water repellant curtain to reduce persistence times within the hospital, especially in areas like the Emergency Department where contamination can occur quickly and frequently with high patient turnover rates. However, as curtain E was the only water repellant curtain examined in this study, more investigation with water repellant curtains both with and without antimicrobial composition would need to be done to confirm this.

For the purposes of resources, the persistence times of $S$. aureus alone were investigated in this study, and samples from the curtains could only be taken every 1-3 days instead of every day. While $S$. aureus is important to study because it is a type of MRSA, it is also a commensal organism that colonizes up to 30\% of the human population (32) and is a leading cause of bacteremia, infectious endocarditis, and device-related infections $(5,28)$. Additionally, there are other important nosocomial infection-causing bacteria to study, including K. quasipneumoniae subsp. similipneumoniae, Acinetobacter baumannii, and Pseudomonas aeruginosa $(1,2,4,23,29)$. These other bacteria may have different persistence times on these curtains than $S$. aureus and may be affected differently by the curtain materials. The persistence times of a variety of bacteria on various curtain types should be measured to determine how different curtain material can affect persistence. Ideally these persistence times would be measured by culturing from the curtain samples every day. Future studies will specifically measure the persistence times of Enterococcus faecalis, Escherichia coli, and $P$. aeruginosa on these different curtain types as these represent three common causes of nosocomial infections $(12,26,31)$ and exemplify three species that contain multi-drug resistant strains including vancomycin-resistant Enterococcus for E. faecalis, extended-spectrum cephalosporin-resistant E. coli, and carbapenem-resistant $P$. aeruginosa (26).

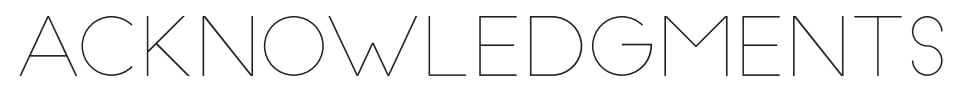

The authors would like to thank Laura Cole for providing the curtain samples used in the testing. 
Staphylococci on hospital curtains $\bullet 61$

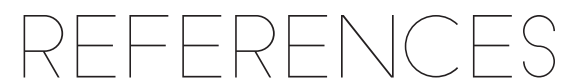

1. Agaba P, Tumukunde J, Tindimwebwa JVB, Kwizera A. 2017. Nosocomial bacterial infections and their antimicrobial susceptibility patterns among patients in Ugandan intensive care units: A cross sectional study. BMC Res. Notes. 10:349.

2. Brasme L, Nordmann P, Fidel F, Lartigue MF, Bajolet O, et al. 2007. Incidence of class A extended-spectrum $\beta$-lactamases in Champagne-Ardenne (France): A 1 year prospective study.J. Antimicrob. Chemother. 60:956-64.

3. Butt IA, Aslam B, Rasool MH, Shafiq HB, Khurshid M, Aslam MA. 2016. Distribution of various pathogenic bacteria from pediatric ward settings. Saudi Med. J. 37:1268-71.

4. Chambers HF. 2005. Community-Associated MRSA - Resistance and Virulence Converge. N. Engl. J. Med. 352:1485-87.

5. Coates R, Moran J, Horsburgh MJ. 2014.

Staphylococci: Colonizers and pathogens of human skin. Fut. Microbiol. 9:75-91.

6. Cockerill FR, Hindler JA, Wikler MA, Patel JB, Alder J, et al. 2012. Methods for Dilution Antimicrobial Susceptibility Tests for Bacteria That Grow Aerobically; Approved Standard. Wayne, PA: Clinical and Laboratory Standards Institute. M07-A9 pp. Ninth ed.

7. Das I, Lambert P, Hill D, Noy M, Bion J, Elliott T. 2002. Carbapenem-resistant Acinetobacter and role of curtains in an outbreak in intensive care units. J. Hosp. Infect. 50:110-14.

8. DeAngelis DL, Khakoo R. 2013. Hospital Privacy Curtains: Cleaning and Changing Policies - Are We Doing Enough? Am. J. Infect. Control. 41:S33.

9. Deurenberg RH, Stobberingh EE. 2008. The evolution of Staphylococcus aureus. Infect Genet Evol 8:747-763.

10. Elliott AG, Ganesamoorthy D, Coin L, Cooper MA, Cao MD. 2016. Complete Genome Sequence of Klebsiella quasipneumoniae subsp. similipneumoniae Strain ATCC 700603. Genome Announc. 4:

11. Gratten M, Battistutta D, Torzillo P, Dixon J, Manning K. 1994. Comparison of goat and horse blood as culture medium supplements for isolation and identification of Haemophilus influenzae and Streptococcus pneumoniae from upper respiratory tract secretions. J. Clin. Microbiol. 32:2871-2.

12. Haque M, Sartelli M, McKimm J, Bakar MA. 2018. Health care-associated infections - An overview. Infect. and Drug Resistance. 11:2321-33.
13. Kotsanas D, Lovegrove A, Sloane TL, Gillespie EE. 2012. Disposable biocidal cubical curtains: Can they prevent the transfer of bacterial pathogens? Healthc. Infect. 17:87-90.

14. Kotsanas D, Wijesooriya WRPLI, Sloane T, Stuart RL, Gillespie EE. 2014. The silver lining of disposable sporicidal privacy curtains in an intensive care unit. Am. J. Infect. Control. 42:366-70.

15. Kramer A, Schwebke I, Kampf G. 2006. How long do nosocomial pathogens persist on inanimate surfaces? A systematic review. BMC Infect. Dis. 6:130. 16. Kurashige EJO, Oie S, Furukawa H. 2016. Contamination of environmental surfaces by methicillin-resistant Staphylococcus aureus (MRSA) in rooms of inpatients with MRSA-positive body sites. Brazilian J. Microbiol. 47:703-5.

17. Larocque M, Carver S, Bertrand A, McGeer A, McLeod S, Borgundvaag B. 2016. Acquisition of bacteria on health care workers' hands after contact with patient privacy curtains. Am. J. Infect. Control. 44:1385-86.

18. Mitchell A, Spencer M, Edmiston C. 2015. Role of healthcare apparel and other healthcare textiles in the transmission of pathogens: a review of the literature. J. Hosp. Infect. 90:285-92.

19. Neely AN. 2000. A survey of gram-negative bacteria survival on hospital fabrics and plastics. J. Burn Care Rehabil. 21:523-27.

20. Neely AN, Maley MP. 2000. Survival of enterococci and staphylococci on hospital fabrics and plastic. J. Clin. Microbiol. 38:724-26.

21. Ohl M, Schweizer M, Graham M, Heilmann K, Boyken L, Diekema D. 2012. Hospital privacy curtains are frequently and rapidly contaminated with potentially pathogenic bacteria. Am. J. Infect. Control. 40:904-6.

22. Rutala WA, Gergen MF, Sickbert-Bennett EE, Williams DA, Weber DJ. 2014. Effectiveness of improved hydrogen peroxide in decontaminating privacy curtains contaminated with multidrug-resistant pathogens. Am. J. Infect. Control. 42:426-28.

23. Schantz M. 2002. Infection control in the home. Caring 21:40-43.

24. Schweizer M, Graham M, Ohl M, Heilmann K, Boyken L, Diekema D. 2012. Novel Hospital Curtains with Antimicrobial Properties: A Randomized, Controlled Trial. Infect. Control Hosp. Epidemiol. 33:1081-85.

25. Shek K, Patidar R, Kohja Z, Liu S, Gawaziuk JP, et al. 2017. Rate of contamination of hospital privacy curtains on a burns and plastic surgery ward: a 
62 - Fine Focus, Vol 52019

cross-sectional study. J. Hosp. Infect. 96:54-58.

26. Sievert DM, Ricks P, Edwards JR, Schneider

A, Patel J, et al. 2013. Antimicrobial-Resistant Pathogens Associated with Healthcare-Associated Infections Summary of Data Reported to the National Healthcare Safety Network at the Centers for Disease Control and Prevention, 2009-2010. Infect. Control Hosp. Epidemiol. 34:1-14.

27. Sood G, Huber K, Dam L, Zenilman J, Riedel S. 2014. A pilot observational study of hydrogen peroxide and alcohol for disinfection of privacy curtains contaminated by MRSA, VRE and Clostridium difficile. J. Infect. Prev. 15:189-93.

28. Tong SYC, Davis JS, Eichenberger E, Holland TL, Fowler VG. 2015. Staphylococcus aureus infections: epidemiology, pathophysiology, clinical manifestations, and management. Clin. Microbiol. Rev. 28:60361.

29. Trilla A. 1994. Epidemiology of nosocomial infections in adult intensive care units. Intensive Care Med. 20:S1-4.

30. Trillis F, Eckstein EC, Budavich R, Pultz MJ, Donskey CJ. 2008. Contamination of hospital curtains with healthcare-associated pathogens. Infect. Control Hosp. Epidemiol. 29:1074-76.

31. Weiner LM, Webb AK, Limbago B, Dudeck MA, Patel J, et al. 2016. Antimicrobial-Resistant Pathogens Associated With Healthcare-Associated Infections: Summary of Data Reported to the National Healthcare Safety Network at the Centers for Disease Control and Prevention, 2011-2014. Infect. Control Hosp. Epidemiol. 37:1288-1301.

32. Wertheim HFL, Melles DC, Vos MC, Van Leeuwen W, Van Belkum A, et al. 2005. The role of nasal carriage in Staphylococcus aureus infections. Lancet Infec. Dis. 5:751-62. 